\title{
Passive Movement and Manipulation
}

\section{By JENNIFER HICKLING, M.C.S.P.}

Passive movement is an established part of physiotherapy and has always been included in our training.

Manipulation occupies a more nebulous position. It is now widely held to be a treatment which the patient should be able to have at any hospital, and that it forms a natural extension of the physiotherapist's job. Physiotherapists do, in fact, make good manipulators, since they have the requisite knowledge of anatomy and joint movement and also preserve an ethical standard.

However, as we all know, manipulation is sometimes considered to lie outside our province. It is curious that this should be so, for manipulation is passive movement; that is, a movement of a joint carried out by an operator without aid of the patient's muscles. Though all manipulation is passive movement, however, not all passive movement is manipulation, and confusion often arises here, as it does over other factors such as definition, indications and effects.

Genuine disagreement exists on all these points. In addition, one tends naturally to view the subject out of one's own experience and practice, which may result in an opinion based on certain aspects rather than on the whole of the matter. Misunderstanding also arises because people are at cross purposes over terms.

I have tried to give a general view showing where the main differences lie and how they can be resolved or, if this is not yet possible, how we can nevertheless work with them. The subject does not divide easily under separate headings, for each point bears closely on others. I suggest therefore that those interested first read the paper through for general argument, and then re-read for particular detail.

\section{DIFFERENCES OVER DEFINITION}

Lack of definition over terms often leads to disagreements which are apparent rather than real, and words often obscure facts. Several ways in which this occurs are listed below.

1. Manipulation is sometimes held to be synonymous with chiropractic or osteopathy because these professions have specialized in manipulation without anaesthesia and did so long before the medical profession. Anyone wishing to manipulate should certainly study their techniques, from which there is much to be learnt.

There is, however, a particular recurring situation which lends more authority to the chiropractor's teaching than it necessarily merits. This occurs when a patient has, say, a pain in the neck that is both suitable for manipulation and quickly curable by it, but the fact is not recognized by the medical profession. After weeks or months of treatment, the patient despairs, "goes round the corner" to the chiropractor and is cured in one or two treatments.

Such an event has two effects. Firstly, to that patient the chiropractor is 100 per cent successful, if not a downright magician. As a layman he cannot be expected to recognise that, though this is a common result of manipulation, it is by no means an invariable one, and that many of the patients before him with similar symptoms might have been no better. Unfortunately it is not only the layman who is overpersuaded in this way, and manipulation sometimes gets a sensational overtone even in the medical world, remarkable results being always expected. This does nothing but harm to its proper assessment as a treatment.

The second result of this story is that, because the patient gets quickly better in the teeth of conventional treatment, the chiropractor's view of what he has done is believed to be correct, which does not necessarily follow. If a man puts out a fire with a gallon of milk declaring that only the animal spirit can conquer the fire spirit, the fact that it has had the desired effect does not mean that his theories are right. This, of course, does not refer specifically to chiropractors, for the point applies universally, and particularly in a field such as this where so much is in dispute. It is very important indeed to be able to see when a statement has really been proved.

2. The physiotherapist often defines manipulation as being a passive movement done under anaesthesia, whereas a passive movement done without anaesthesia is called forced or relaxed passive movement. This will not do, since many experts in manipulation do not use anaesthesia.

3. The word is sometimes reserved for mobilising a movement not normally under voluntary control, such as the gliding movements at the wrist. This again is a definition that is not used by many experts.

4. It is not satisfactory to reserve the word for movement carried out in a painful, but not a painless, range, for manipulation of a joint often involves doing both.

5. A common view is that "manipulation" is a short quick movement, while "passive movement" is slower and more gentle. This distinction cannot be pressed too far; for instance, when treating a cervical disc lesion, especially with gross painful limitation of movement, one often begins with very gentle delicate movements while trying to establish which manoeuvre will be helpful. Once this has been determined and the patient has begun to improve, the same manoeuvre may well be carried out much more firmly, quickly, or with more force. It would generally be agreed that the first manoeuvres were passive movement and the last ones manipulation, but exactly where one merges into the other is hard to define and the effort makes for some very unreal distinctions.

6. Dr. Cyriax, in his article in this journal, defines manipulation as passive movement done for one of two purposes: to rupture an adhesion or to reduce internal derangement. That is, it is defined by the disorder and not by method. This sometimes leads to difficulty for the physiotherapist, since the medical profession is not agreed about diagnosis and the same signs and symptoms may be ascribed by different doctors to different conditions. The way out of this difficulty is discussed below.

7. It must also be remembered that there are several terms, such as mobilisation, forcing, stretching, as well as passive movement and manipulation, all of which may be used by different people to mean virtually the same thing; this sometimes gives rise to what sounds like disagreement when in fact there is none.

The physiotherapist wishing to study or contribute to this field has to pick her way through the above points and must always make a meticulous effort to define terms and establish what are the basic facts under discussion.

One definition of manipulation is "the handling of objects for a particular purpose", and if "joints" is substituted for "objects" it gives a very fair picture of the procedure: a passive movement done with anything from great delicacy to considerable force, and with varying range, speed and purpose. I think myself that research into the subject is only harmed by drawing a hard line between passive movement and manipulation, for they can only be usefully studied together; it would, in fact, be helpful to drop the term manipulation altogether, were this not certain to be con- 
strued as implying that it lay outside the sphere of physiotherapy. They are considered here as part of one subject. Either term is used as it possesses most nearly the required meaning.

\section{DIFFERENCES OVER DIAGNOSIS}

There are three main categories of disorder which are agreed, by one expert or another, to respond at times to passive movement. These are: arthritis, adhesion, internal derangement.

However, the medical profession does not agree, especially at the spine, on which signs and symptoms indicate a particular disorder nor upon when passive movement should be used. The physiotherapist, knowing that manipulation has dangers and that doctors disagree, may well say out of a sense of responsibility to the patient "this technique should not be used by us until the medical world is agreed about it".

This is a pity for two reasons. Firstly, many patients are deprived of effective treatment, and though this would be justifiable if such treatment meant subjecting the patient to danger, I do not think this need be so, as I hope to show.

Secondly, it is the critical use of the techniques themselves that can do so much to clarify the disputed points. The search for an agreed, definitive diagnosis should, of course, go on, but if the physiotherapy profession waits for it, we are likely to wait a long time. We can, in the meantime, use the techniques, study methods and effects, and contribute a mass of evidence to the central discussion.

Danger in this situation is avoided by following the precept that manipulation is controlled not by the diagnosis but by the patient's signs and symptoms. These are not in dispute; they are verifiable and re-verifiable, and it is to them that treatment is pinned and by them that it is regulated. This point is enlarged upon under "Danger and its avoidance" below.

\section{ARTICULAR PATTERNS}

There is no attempt in this paper to discuss examination in detail or describe individual lesions in particular.

The difference of opinion about these disorders, however, means that discussion of manipulation can usually be kept clear only by reference to the physical findings rather than the diagnosis. Articular signs are therefore discussed here briefly both to help define the conditions in question, and because painful limitation of movement is one of the main problems with which passive movement hopes to deal.

(Further discussion of signs and symptoms, insofar as they control treatment and are used in reassessment, takes place under "Treatment" below.)

\section{Selective Tension}

Dr. Cyriax's method of examining the moving parts by selective tension must be referred to here, to settle terms of reference. Anyone wishing to study it further is referred to his textbook on the subject ${ }^{1}$.

Tension is put on each of the tissues in turn to see which test provokes pain, or is otherwise abnormal. For this purpose the tissues are divided into the contractile and inert groups.

The contractile group; comprises muscles, tendons, and their attachments, and is so-called because tension can be put upon them by making them contract. The test for them is a strong static contraction. The examiner holds the joint in a neutral position so as to avoid tension upon the inert tissues as far as possible and to prevent movement. The muscle group is then tested in isolation by asking the patient to contract.

Referred (and indeed quite normal) tenderness is very common and misleading. Tenderness in a muscle is only accepted as arising from that muscle if it is found to be painful when tested in the above way. This principle is applied to other tissues as well; the tissue at fault is isolated by selective tension and only when it has been named does palpation for tenderness, confined to that tissue, play a part in localization, and then only if the structure is fairly superficial.
The inert group comprises all other tissues, and is tested by being stretched or pinched: that is, by passive movement done through the full possible range.

Active movements can also be used to test the inert tissues and are often more convenient, but a small passive push should be added at the end of movement to see what the extreme of possible range feels like, to see if pain is provoked, and to judge muscle spasm.

An active movement does, of course, involve muscles as well, but while it is a good guide to the state of the inert tissues about a joint it is a very poor guide to the contractile tissues. For instance, if a patient can lift his arm easily to full elevation one can be fairly sure that there is nothing wrong with the joint but not at all sure that there is nothing wrong with the tendons about it. Painful limitation of an active movement, therefore, may generally be taken as indicating trouble with the inert tissues; if there is any doubt, the tissue groups can be tested separately by passive and resisted movements and the findings weighed against each other.

\section{Articular Patterns}

Examples of the three basic conditions under discussion which may require treatment by passive movement are as follows:

(a) Arthritis or capsulitis. (The term "capsulitis" may be used here to describe that aspect of the condition which responds to passive movement.) The painful limitation of movement at a joint following immobilisation or trauma; painful contracture accompanying osteo-arthritis at the cervical spine or hip joints.

(b) Adhesion. Post-traumatic adhesion giving rise to persistent discomfort with minor limitation of movement, and preventing full return of function after athletic injury, fracture, or operation.

(c) Internal derangement. Disc lesions at the spine, or derangement, or nipping of the synovial fringe, of the facet joints. (This is where one of the main arguments about diagnosis occurs.) Loose body in the elbow or knee.

If any of the above conditions are present there will be, in general, painful limitation of passive and active movements, and the static resisted movements will be painless. This painful limitation of movement will fall into either the capsular or the non-capsular pattern.

1. Capsular pattern. This is found in, and is diagnostic of, arthritis or capsulitis ${ }^{\mathbf{1}}$. ALL movements are painful and limited in the capsular pattern peculiar to that joint.

The capsular pattern is constant for any one joint, in that the loss of range in one direction is proportional to the loss of range in the others; the severity varies, but the all-over pattern does not. For instance, at the knee the pattern is a little loss of extension, a gross loss of flexion and about equal loss of rotations; if the arthritis becomes worse, movement is lost in all ranges proportionately to each other and the all-over pattern is preserved. This is, of course, quite different from the pattern of arthritis at the hip or cervical spine, but is similar for all other arthrites at the knee.

2. Non-capsular pattern. This is present both with adhesion and with internal derangement (which can mimic each other closely), and consists generally in some movements being limited and painful and others not; i.e. not the whole of the joint.

With an adhesion, those movements which move or stretch the lesion will hurt while those that relax it will be full and painless.

With internal derangement the pattern is entirely variable and arbitary and may involve gross painful limitation of movement in several directions or merely a little pain at the extreme of one range. All that can be said is that the pattern will not be the capsular one.

X-ray

An X-ray may be essential to exclude serious disease or injury. X-ray appearances of osteo-arthritis or diminished joint space, however, are only accepted as relevant to the 


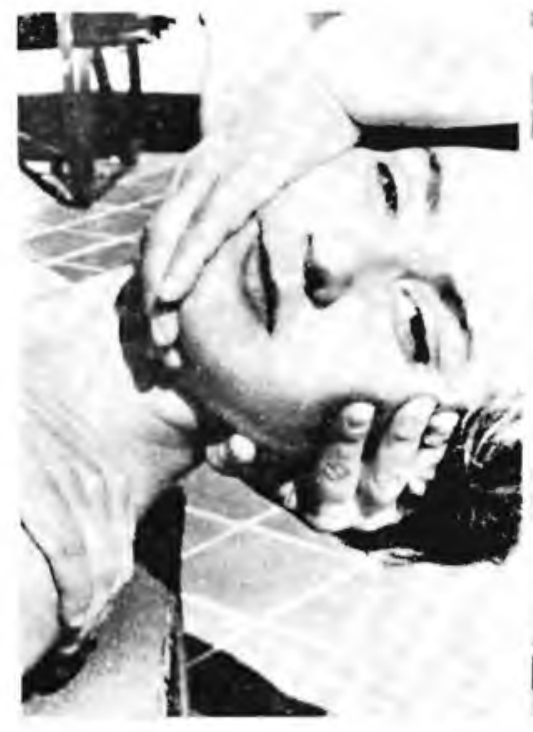

(a) Positioning.

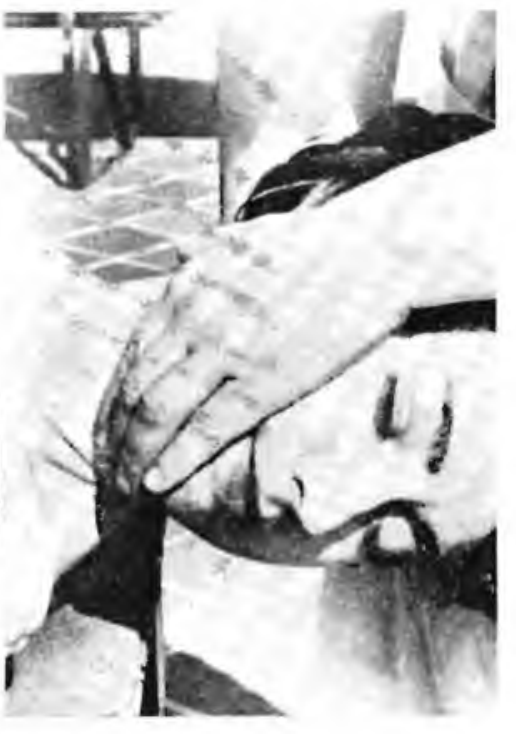

(b) Execution.

Fig. 1 Close-up of rotation at the cervical spine.

patient's pain if they are borne out by clinical examination.

For instance, it is quite possible for a patient to have marked cervical osteo-arthritis on the X-ray, but on clinical examination to have a non-capsular pattern of sudden onset; i.e. it is not his arthritis as such which is causing the pain. Manipulation of such a neck should be for the noncapsular pattern and of the "not set" variety (discussed below); to regard the condition as arthritis and manipulate it as such is likely to be ineffective, if not harmful.

The decision to manipulate, therefore and the nature of the lesion being treated is based primarily upon clinical findings.

\section{TREATMENT BY PASSIVE MOVEMENT OR MANIPULATION}

"Set" or "Not Set"?

This is one of the crucial distinctions that has to be drawn in manipulation.

Where there is arthritis or adhesion, the procedure is "set", in that the operator knows in advance which range of movement will be pushed during treatment. Certain movements are found on examination to be limited and painful; once it has been decided to try passive movement, it will be in those directions that the joint must be moved to stretch or rupture the contracted or adherent fibrous tissue. There are still other decisions to be made, about range and force and speed of movement, but the direction is predetermined.

Internal derangement presents a different problem. Here one cannot predict the effective manoeuvre with certainty, for one is trying to shift a mechanical block and it is not possible always to know what will achieve this. It may be movement into a painful range, or a painless range, or both. It may be movement in one direction only, or several. It may be possible to make the condition better with one manoeuvre and worse with another. The correct direction of thrust has therefore to be discovered, as well as range and force and speed. The manipulation is therefore called "notset", and the procedure is as follows.

Firstly the joint is examined to determine the relevant signs and symptoms. A manoeuvre is then tried, and the joint is re-examined to see what changes have occurred. If there is a good result, it is repeated; if there is no change, another manoeuvre is attempted. The joint is then once more examined to see the results of the second manoeuvre, and the selection of the next one is based upon that examination. If there is any suggestion of worsening, another direc- tion may be tried with caution or it may be decided to abandon manipulation. The manipulator thus feels his way throughout the treatment, regulating it by any changes that occur. In no sense does the manipulation have to be symmetrical and may consist in one manoeuvre repeated many times.

Adhesion and internal derangement both give rise to the non-capsular pattern and it is often difficult to tell them apart. Indeed at the spine medical opinion may be far from agreed over the meaning of a syndrome. For example a unilateral pain in the back, giving rise to pain in some directions and not in others and with no other physical signs may be attributed to internal derangement or to an adhesion. The history may give a clue (for example, if the pain came on suddenly it is not likely to be an adhesion), but sometimes the physiotherapist has no clear directive, though it would commonly be agreed that this is the sort of pattern which responds to manipulation.

The proper manipulation for an adhesion is a short quick movement of sufficient force to rupture the limiting fibrous tissue, but such a procedure should not be used unless the physiotherapist has a clear diagnosis and the doctor's backing. If there is a possibility of internal derangement it is always best to be on the safe side, and adopt the procedure for "not set" manipulation as above. This is, in essence, to feel one's way for direction as well as for range and force, and to observe results at re-examination before attempting more.

\section{Anaesthesia}

It is not possible to follow the above procedure on the anaesthetised patient, and in Dr. Cyriax"s view anaesthesia is strongly contra-indicated in "not set" manipulation where it is impossible to predict with certainty the effective manoeuvre and also possible to make the patient worse by doing the wrong one. It is claimed that the total relaxation results in manipulation being more effective; this is indeed true and whereas useful if the right movement is done, if the wrong one is chosen real harm can result. It is always possible to get sufficient relaxation on the conscious patient, and this is discussed further below.

On the other hand anaesthesia is not always contraindicated in "set" manipulation, and may be necessary if pain and muscle spasm otherwise prevent adequate joint movement. Even so, it is not always useful. If gross limita- 
tion of movement is forced, the joint sometimes responds with a marked after-flare. The physiotherapist's struggle to maintain range while this is subsidising may amount to much the same thing as trying to achieve it more slowly on the conscious patient. If manipulation under anaesthesia is carried out, intra-articular hydrocortison given 24 hours beforehand does much to abate this reaction.

\section{Note-keeping and Co-operation with the Doctor}

Good note-keeping contributes enormously towards the proper use of passive movement and is vital for its safety and for proper research.

The treatment card must have sufficient space to write an adequate history and all signs and symptoms relevant to treatment, so that it gives a clear picture of the problem with which physiotherapy is trying to deal. If these details are not taken down at the clinic, time must be made at the first visit for such an examination. If as is usual, there is no time, then it is not only justifiable, but is in the best interests of the patient, for the whole of the first treatment to be given up to this purpose.

There must also be room on the card for changes in physical signs to be noted at regular reassessment. Such notes may be brief in "set" manipulation where the treatment is often a routine one and follows from the original examination. In "not set" manipulation, on the other hand, particularly at the spine, notes have to be kept much more fully, since individual manoeuvres, and the changes resulting from them, often have to be written in detail.

Close co-operation with the doctor is essential and the treatment card will often help here.

In the first instance, the physiotherapist should be careful not to manipulate except at the express wish of the doctor in charge; "physio. please" is not sufficient. This is partly because not all doctors agree to physiotherapists manipulating, and partly because there are dangers which have to be excluded before manipulation is used. This returns us to the problem of where passive movement becomes manipulation, since the first is an unquestioned part of our techniques and the latter is not. Many doctors would not hesitate to let us use the former on our own judgment, while feeling strongly that this is not sufficient for the latter. This impasse can be resolved by the responsibility of the physiotherapist towards the doctor's wishes and discussion with him so that he appreciates the problem and can give guidance.

There should also be close co-operation with the doctor while treatment is going on, so that its effects, successes and failures are always under review.

Here various actions will help. It is useful if a physiotherapist can be at the clinic when the patient is put onto treatment, so that its aims and possibilities can be discussed. The treating physiotherapist should also have ready access to the doctor for guidance and discussion if difficulties arise during treatment. Finally it is useful if the patient sees the same doctor at follow-up visits. I know that many other factors have a bearing upon whether this is either possible or advisable. Nevertheless, where this is not the practice the doctor sometimes has little idea of the real effects of physiotherapy, and an important corrective to ineffective treatment is lost. If the same doctor or physiotherapist cannot be present at the follow-up visit then at least the treatment card should be there. It should be clearly understandable by the doctor (an important point) and accompanied by a note from the physiotherapist giving her view on progress. In this way a real effort can be made to stop treatment if it does not help, which is one of the best things that can be done for physiotherapy.

Should a patient be cured quickly by manipulation, he is often discharged clerically from the physiotherapy department without reference back to the doctor; this is done to save time in crowded clinics. However, the fundamental important time-save is that treatment is applied where it works and abandoned where it does not. If a treatment is quickly successful, this should at least be brought to the doctor's attention. The patient need not necessarily return to the clinic; the physiotherapist can merely have a word with the doctor, showing him a treatment card which gives the relevant signs and symptoms. His memory is thus refreshed about the syndrome that was treated and he can build up a clear picture of when a treatment works.

\section{Signs and Symptoms, and Assessment of Progress}

Treatment by passive movement, particularly manipulation, usually results in steady progress from the beginning. If this does not occur, the position should be reconsidered.

The conditions under discussion give rise to certain signs and symptoms which are relevant in assessing progress. They are now discussed under three headings and the way in which they can be used for this purpose is discussed.

\section{Pain}

The more severe the lesion the more distal and extensive will be the reference of pain and vice versa? ${ }^{\text {. The position }}$ of the pain is therefore very important, and is perhaps the most valuable single factor in guiding treatment and avoiding danger, especially at the spine. If a manoeuvre shortens the pain or moves it proximally, this is an encouragement to continue. Conversely, if a manoeuvre moves the pain more distally, that particular manoeuvre is likely to be harmful, and if the same effect is met in several directions, manipulation should not be continued.

The physiotherapist must ask the patient to say if the pain changes in this way while the joint is being moved into position, and if the change is an adverse one, that manoeuvre is not pressed home (i.e. there is positioning but not execution). Response is thus assessed before the final thrust and guides whether it is given or not. In this way, the worst that occurs is a little temporary aching; such control cannot, of course, be maintained under anaesthesia.

Other aspects of pain, such as its severity, duration or ease of provocation will also often help in assessing progress.

\section{Articular Pattern}

Firstly, there is pain and limitation of movement in the capsular or non-capsular pattern. Secondly, there may be deformity, and thirdly there may be signs of inflammation.

The last two can be dealt with fairly briefly here. Marked deformity of this kind often accompanies internal derangement at the spine, and may call for certain precautions. It is not a contraindication to passive movement, but often causes difficulty in handling the joint. The aim of treatment is of course to abolish it, and a manoeuvre that tends to do this is a useful one. Signs of inflammation (such as heat, fluid in the joint, etc.) are again not in themselves contraindications to treatment, though in conjunction with other findings they may be so, or may call for a cautious approach. Their diminution from one treatment to the next, e.g. with a loose body in the knee, may be a helpful guide.

Pain and limitation of movement at the joint require rather more to be said about them here.

With adhesion or internal derangement, the aim of passive movement is usually to achieve a full and painless range of movement by breaking the adhesion or reducing the displacement. Results are quick if they are achievable; the patient is well in 1-5 treatments. An increase in painless range obviously indicates improvement; assessment is from one treatment to the next with an adhesion, and occurs many times in one treatment for internal derangement, for reason already discussed.

There is no good and may be harm in continuing manipulation after a full and painless range of movement has been achieved (a view not held by some chiropractors). For instance, when derangement has been reduced, continued joint movement merely causes unwanted mobility and may cause re-displacement.

In capsulitis the aim of treatment may again be the restoration of full painless movement, where the condition is superimposed on an otherwise normal joint, such as in immobilisation or traumatic capsulitis. In osteo-arthritis, on the other hand, where the capsulitis occurs in a joint with irreversible degenerative changes, such a result is not 
necessarily hoped for. Prolonged, fairly strong stretching is often extremely successful in such joints, but the main hope is to get a good symptomatic relief lasting many months. If movement also improves, so much the better, but this is not the criterion of successful treatment. It is quite possible, for instance, for a patient to have the pain from her cervical osteo-arthritis almost totally relieved while still having gross limitation of movement.

The physiotherapist has to judge what is possible. One must not continue to manipulate for an increased movement or a relief of pain which are not achievable. It is no good trying to reassess too often and once a week is usually about right (treatment being two or three times weekly). The patient often finds it difficult to judge progress, but can generally make a decision if asked to compare his present comfort with that of a week ago. It is worth continuing treatment if he says confidently that he is much better; if he is uncertain, and this bears out one's own view, treatment for one more week may be tried, but with the same result again it should then be stopped.

\section{Extra-articular Signs and Symptoms}

These occur where the disorder is of such a nature as to involve tissues outside the joint, such as in a disc lesion which is compressing a nerve root. The most important is painful limitation of straight-leg raising, which provides a very exact guide to progress, and where increase in range can usually be taken as an indication to repeat the manoeuvre that produced it. Other findings include painful twinges, pain on coughing or sneezing, and so on. Obviously the aim of treatment is to abolish them, and they can be useful in determining whether to repeat or change a manoeuvre.

\section{THE AVOIDANCE OF DANGER}

There are dangers in manipulation as in any potent treatment, and no attempt is made here to deal with the special precautions which apply to individual disorders. Nevertheless, I think something useful can be said in general terms about these hazards, which naturally cause many people concern.

Two main rules can be used, either together or singly, to govern the safe use of passive movement. These are:

Rule 1 . Only go on to the next manoeuvre when the results of the last one have become clear.

Rule 2. Only increase force when it is clear that rather less force in that direction has already had good effect.

The first rule summarizes the procedure for "not set" manipulation already described. Both rules are referred to again below, in considering how danger is to be avoided.

Danger arises if there is error at any one of four points:

(1) Selection of the case.

(2) Selection of direction of force ("not set" manipulation).

(3) Selection of amount of force.

(4) The way force is used.

The errors that may occur at each point, and the way in which they can be avoided, are considered in turn.

\section{A. Unsuitable Case}

Medicine is not an exact science, and no one would claim that it is possible to be 100 per cent correct in all diagnosis. Again, as already discussed, the medical profession is far from agreed over diagnosis in these conditions, and where there is disagreement someone must be wrong.

Argument over diagnosis lies, of course, largely within the doctor's province, and there is no attempt here to enter it. However, as a result of the argument it is sometimes held to be dangerous to manipulate until the disagreements are resolved. It is this view which is discussed here, for it colours the whole subject of manipulation and profoundly influences its use.

I would suggest that manipulation can nevertheless be used if it is recognized that its control is by the signs and symptoms of the disorder, rather than by the diagnosis.
Herein lies safety. This point was made earlier and is now dealt with in greater detail.

In any lesion there are certain physical findings; these establish the diagnosis; upon the diagnosis, the decision to manipulate is based. The manipulator, however, guides his treatment by constant reference to the original signs and symptoms, as already described. It is changes in the signs and symptoms which determine whether he stops, goes on or varies treatment. Even when the diagnosis is unquestioned, this remains true. The diagnosis and examination of internal derangement at the spine, for example, can do no more than say "this looks as if it is suitable for manipulation". Whether it is, and whether manipulauion will succeed, can only be decided by the attempt, the guiding factor being the physical findings.

In a sense, therefore, the diagnosis can be dispensed with. Obviously precision in diagnosis makes for precision in treatment, but its absence or error need not make for danger or compel inaction. It is quite possible to manipulate a joint responsibly and safely, and at the same time consider which of several disorders might give rise to the syndrome present, and which is suggested most strongly by the changes that occur. I would submit that the willingness to do this can contribute a lot of useful evidence to the argument over diagnosis.

\section{B. Wrong Direction}

There is little danger of error here in "set" manipulation, since those movements which are painful and limited at examination are those which have to be pushed.

In "not set" manipulation for internal derangement, on the other hand, "error" cannot always be avoided, for the manipulator has to discover the right direction. It is only finding a manoeuvre ineffective or exacerbating that enables one to discard it in favour of another. Such "error" however, will do no harm provided it is subject to the two rules set out above; that is, firstly, that it is controlled by the method of reasssessment already described, and secondly, that too much force is not used too soon, a point now dealt with.

\section{Too Much Force}

This is where real danger lies; error in other ways only becomes dangerous if added to error here.

"Too much force" may be any force at all on the wrong case or in the wrong direction. If case and direction are both suitable, "too much force" is still possible, though it is likely then to result not so much in danger as in severe, avoidable treatment soreness. "Too much force" cannot be measured by a gauge, and requires judgement and a balance between courage and restraint in the manipulator.

Rule 2 above sets out the principle to be followed here, and the method is now described in greater detail.

A preliminary push is applied and the results are observed at re-examination. If there is a good effect, more is done. If this results in further improvement, real firmness may then be used. If there is any suggestion of adverse effect, that manoeuvre and possibly the whole question of passive movement, may be reconsidered. The result may be assessed several times in one treatment for internal derangement, or from one treatment to the next, as is more usual in capsulitis and adhesion.

Where the condition is acute or one is unsure of one's ground, the first attempt may well be so gentle as to be almost useless. It is only the emergence of some slight improvement that encourages the manipulator to continue, and probably only by the second or third attempt that he uses requisite force. Experience naturally lends speed here.

Provided the use of force is always subject to this rule, no one need be afraid of it, and the manipulator may often find herself using, and being required to use, considerable strength.

The inexperienced and cautious probably begin by using insufficient thrust. It is much better for the patient and for the course of manipulation to be on the safe side in this way, but to avoid disappointment they should remember that it is only after ten patients are no worse that their treatment 


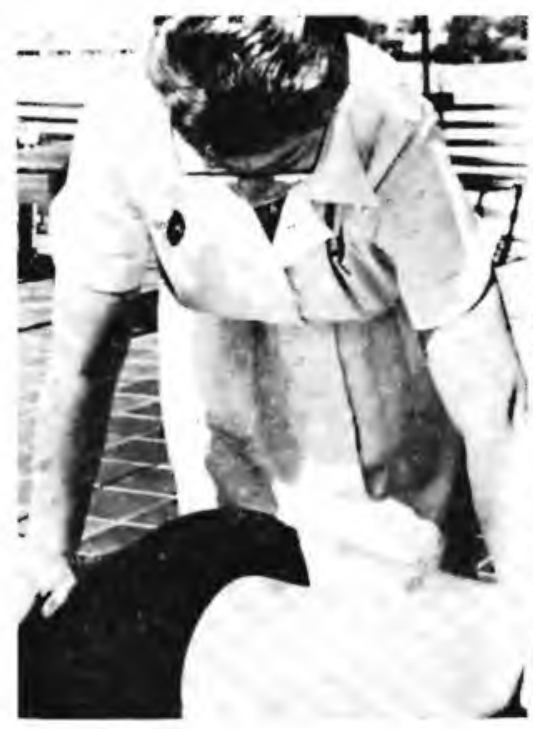

(a) Positioning

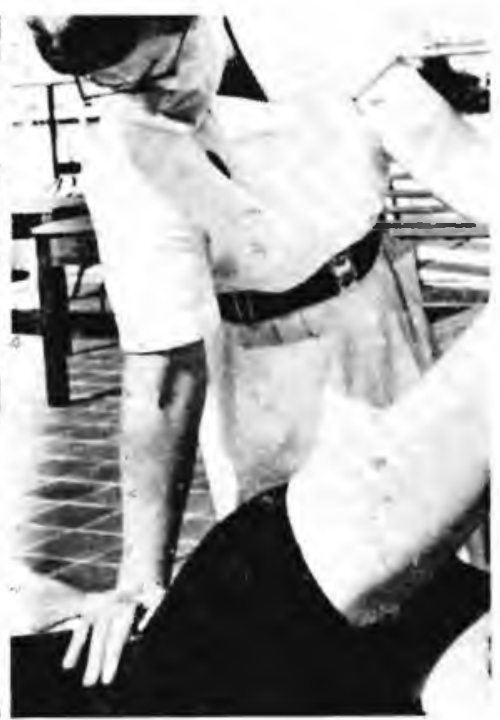

(b) Execution

Fig. 2 Rotation in Extension at the lumbar spine.

may become confident enough to make the next patient better.

There remains a situation, which occurs only seldom but regularly for the manipulator, when preliminary manoeuvres produce no change at all. The physical signs and symptoms suggest the case to be suitable for manipulation, but clearly the only way of achieving any change is to use considerable force, and then perhaps find, too late, that it is an adverse one. Whether this should be done is a matter for individual opinion, and various factors have to be considered such as the gravity of the condition and the possibility of other treatment helping. Certainly no one without experience should take this step, and no physiotherapist should do so except at the express wish of the doctor and in consultation with him.

\section{Traumatic Use of Force}

Even if the case is suitable and direction and degree of force correct, the manoeuvre can still be done traumatically. Manipulation then becomes unnecessarily painful and alarming for the patient and causes excessive treatment soreness. If there is also error at any of the other points, the result will be proportionately worse.

This subject is intimately connected with technique, and indeed is technique, and is therefore discussed with other matters under this heading.

\section{TECHNIQUE}

A good technique is compounded of many factors bearing upon each other.

It is important to avoid "taking a run at the joint". This is sometimes done to get a quick forceful movement and is a common cause of treatment soreness. Movement becomes uncontrolled at the extreme of range; it therefore becomes unnecessarily painful, harmful, frightening to the patient, and associated with increased muscle spasm. The same movement done in a controlled way has none of these effects.

This is part of the skill that the manipulator must acquire: the ability to apply an exact amount of force an exact range of movement, without uncontrolled "run up" to the range or "over run" beyond it. Such skill is always important. It is especially so when it is decided to move a joint into a range of movement that is grossly limited, since it is best to do this fractionally; that is, to move through a few degrees first, observe results, and then move further.

It is helpful here to think of each manoeuvre as having two parts: positioning and execution.

Positioning is moving the joint up to the extreme of possible range with the patient trustful, relaxed and as comfortable as possible, and oneself in a good functional position. Execution is the final effective over-pressure, once proper positioning has been achieved. (Not all manipualtion involves movement into an extreme of range, but this picture can be used to express an important principle in technique.) Judgement in execution is very important, but the main art of manipulation probably lies in positioning, and it is this which is chiefly responsible for relaxation.

At least three factors have a bearing here. Firstly, if the patient is frightened he will be unable to relax, and a simple explanation of what one is attempting will do much to set him at ease. Secondly, if the patient is not used to full joint movement (for one of many reasons such as being unathletic, sedentary, aged, etc.) the sensation of having the joint taken to extreme of range may well be disturbing, at least, for him and he may again be unable to relax. Finally, an acute condition may well cause pain not only at the end of possible range but on movement from one position to another; positioning will have to be slow and delicate to minimize both this and the consequent tightening of muscles.

Forceful attempts to overcome any of these factors will produce more pain and muscle spasm in a vicious circle that achieves nothing except the patient's strong disinclinication to be manipulated again. A joint cannot be browbeaten into co-operation any more than a strange child or a wild animal. It is patience and subtlety that count, and, if necessary, more time must be taken over positioning. The second treatment will often take a fraction of the time of the first because the condition has improved and the patient is no longer apprehensive.

It is always possible to get relaxation sufficient to achieve what one wants, and muscle spasm is valuable in indicating the state of the joint and guiding what one does. Where it cannot be overcome it may well be inadvisable to try; an attempt to force such a movement should be considered very carefully indeed. This is another warning that is removed by anaesthesia.

Stancc and grip must be economic in effort for the physiotherapist and comfortable for the patient. A good technique stems from first considering what strain one wishes to apply to the joint, then studying how various experts have achieved this, and finally adapting these findings to one's own physique and aptitudes. 
The hand should at all times be a perfectly comfortable instrument for transmitting force. It moulds itself to the patient and is both gentle and confident. One must be able to apply considerable force without passing on a sense of effort to the patient by unnecessary tightening of the fingers.

The patient will sense any lack of authority in hands or manner and find it difficult to relax. There is, unfortunately, no way out of the fact that one practises on one's first patient and indeed probably on the first hundred. What one practises however, must be how to reduce a disc lesion and not how to do a rotation.

The manoeuvres themselves must all have been practised many times on many models, so that one is familiar with the wide variety of joint movement in different physiques, and what the extreme of range feels like to oneself and to the model. There is no need for such practise to be forceful; in fact it is better for it not to be. The aim is to study grip, handling, positions and stance.

Thus when first treating a patient one can at least handle the joint adroitly and is thus able to concentrate on the lesion, exploring range and muscle spasm and results with hands and observation.

\section{PAIN AND TREATMENT SORENESS}

Pain has to be considered in two ways. We have already seen how its behaviour can be used as a guide to assessing progress. Secondly, there is the question of how much pain is inseparable from treatment, and this is now discussed.

To push a painful range of movement is sometimes forbidden as dangerous, but I hope I have said enough to show why this need not be so. Passive movement for capsulitis or adhesion must at least hurt a little, since it involves pushing the painful and limited range. The effective manoeuvre in internal derangement may be in a painful or a painless range and one has to discover which it is. To forbid painful movement altogether is to prevent treatment where it can be most effective.

Obviously, movement into a painful range must be subject to what the patient can reasonably tolerate and it is unwise and inhumane to exceed this. That having been said, however, it must also be said that the criterion of whether or not a manoeuvre should be used is not "does it hurt?" but "does it have the right effect?'. The method of reassessment that decides this and the rules which prevent exacerbation have already been discussed.

Manipulation is unfortunately sometimes made unnecessarily painful by bad technique. Various points to be avoided have already been mentioned under "Treatment"; they can be eliminated by skill and care, and a mental projection of oneself into the joint one is treating. Treatment soreness does occur, however, and must be reckoned with. Treatment should stop before it becomes marked, blurring the pattern on reassessment which is the manipulator's guide.

A point to remember here is that if, during manipulation, several menoeuvres are tried without effect, treatment soreness can rapidly become severe; the original condition is as painful as ever and the joint has now been moved about a good deal. Once a successful manoeuvre is found, the original pain from the lesion markedly subsides and treatment soreness is then swallowed up in the general improvement.

The onset and severity of treatment soreness is in any case exceedingly diverse, varying with the lesion and the sensitivity of the patient's physique to joint movement. Treatment at one session continues until the required effect has been produced, or until either joint or patient has had enough.

\section{EXERCISES}

(This paper deals with lesions of the inert tissues where there is a normal neuro-muscular system. Some of what is now said applies when neuro-muscular disorder is present, but other factors may then take precedence.)

There is sometimes a difference of opinion about the treatment of these conditions, and exercises may be used instead of, or with, passive movement. This raises various points which are now discussed.

If a lesion requires treatment by joint movement, it is of course theoretically possible to make the movement an active one (exercise) instead of a passive one (or manipulation), the patient's muscles being used instead of the physiotherapist's. Both procedures may have the right effect and both of them can therefore be found useful in the same condition.

Exercises may therefore be used to produce a joint movement, the movement itself being the effective measure for the inert tissues and the muscles merely being used to attain it.

Exercises may also be used in these conditions because it is considered that the muscles themselves require treatment. For example, it may be felt that strengthening or training the muscles about a joint which is degenerate or liable to derangement will render it less liable to strain or redisplacement. Here the exercise is directed primarily to the muscles, and the joint movement is subordinate to it.

It is not always kept clear, however, that a movement which is nicely calculated for a particular effect on the muscles may be excessive or inadequate or in the wrong direction for the primary lesion of the inert tissue. For instance, free active exercises for the muscles about an arthritic joint may result either in the joint flaring or becoming more and more stiff if the movement is too drastic or insufficient for the capsulitis itself. Again, once reduction of internal derangement is complete, emphasis should be on using the joint in good positions only and exercises for the muscles must be subject to this and avoid movement which may upset the lesion.

It is sometimes held that exercises are safer than passive movement because the patient will stop short of doing himself real harm and thus establish an automatic control; this is often the view of those who fear that if the physiotherapist is turned loose with passive movement, anything may happen. It is possible for free active exercises to be far more traumatic than proper manipulation, however, especially if the patient gets hold of the pernicious (in internal derangement) idea that the more it hurts the better it is. It is also difficult, in practice, to use them to produce the selective movement required for internal derangement, where direction of thrust is based upon, and varied by, constant reexamination.

Lastly, range of movement and degree of force must always be under control. Passive movement is much more efficient here; the physiotherapist's hands feel what is being done and also govern it more exactly. Sometimes it is not possible for the patient himself to get the necessary force applied to a particular range without "taking a run at it", which, as we have seen, is one of the main causes of needless trauma. The physiotherapist can get better leverage, knows where the movement should stop, and can control it properly.

Assisted, resisted, hold-relax techniques overlap passive movement much more closely, and can often be used with exactly the name effect. The physiotherapist has her hands on the patient and can assess and control precisely what is happening.

Use of the patient's muscles, however, must never distract attention from the inert tissues that are the site of the lesion. If movement is to be the curative measure, it must be subject primarily to the needs of those tissues and only secondarily to the needs of the muscles.

\section{Summary}

The use of manipulation by physiotherapists is considered as part of the use of passive movement. Differences in definition and over diagnosis are discussed. It is suggested that manipulation may be used safely and with good effect in spite of these differences, and that its critical use can do much to resolve them. Medhods whereby this can be done are put forward, and general points about technique and exercises are discussed.

\section{REFERENCES}

1. Cyriax, J. (1962). Textbook of Orthopaedic Medicine, Vol. 1. 